\title{
Magnetic moment investigations of epitaxial magnetite thin films
}

\author{
M. Kallmayer, ${ }^{1}$ K. Hild, ${ }^{1}$ H. J. Elmers, ${ }^{1}$ S. K. Arora, ${ }^{2, a)}$ Han-Chun Wu, ${ }^{2}$ R. G. S. Sofin, ${ }^{2}$ \\ and I. V. Shvets ${ }^{2}$ \\ ${ }^{1}$ Institut für Physik, Johannes Gutenberg-Universität Mainz, D-55128 Mainz, Germany \\ ${ }^{2}$ Centre for Research on Adaptive Nanostructures and Nanodevices (CRANN), School of Physics, Trinity \\ College Dublin, Dublin 2, Ireland
}

(Presented on 8 November 2007; received 12 September 2007; accepted 4 December 2007; published online 13 March 2008)

\begin{abstract}
In this report, we provide an $\mathrm{x}$-ray magnetic circular dichroism (XMCD) study for $100 \mathrm{~nm}$ thick epitaxial magnetite $\left(\mathrm{Fe}_{3} \mathrm{O}_{4}\right)$ films on $\mathrm{MgO}(001)$ and $\mathrm{Al}_{2} \mathrm{O}_{3}$ (0001) substrates. For XMCD, we recorded the surface sensitive total electron yield and the bulk sensitive transmission spectra. From the analysis of the XMCD data, we find an increased Fe spin moment (10\% larger) at the surface of the film on $\mathrm{MgO}$ (100) with respect to the corresponding bulk value of the film. Surface and bulk spin moments of the film on $\mathrm{Al}_{2} \mathrm{O}_{3}(0001)$ are almost equal. For both films, the bulk orbital to spin moments ratio increases from zero at $70 \mathrm{~K}$ to $0.03-0.04$ at $300 \mathrm{~K}$. For $\mathrm{Fe}_{3} \mathrm{O}_{4} / \mathrm{MgO}$ (001), the surface orbital to spin moment ratio behaves similarly to the bulk value, while the orbital to spin moments ratio is increased at the $\mathrm{Fe}_{3} \mathrm{O}_{4} / \mathrm{Al}_{2} \mathrm{O}_{3}$ (0001) surface to 0.06. The observed differences between films grown on $\mathrm{MgO}$ and $\mathrm{Al}_{2} \mathrm{O}_{3}$ are explained within the framework of differences in mismatch strain experienced by the films. (C) 2008 American Institute of Physics.
\end{abstract}

[DOI: $10.1063 / 1.2838982]$

\section{INTRODUCTION}

Magnetite $\left(\mathrm{Fe}_{3} \mathrm{O}_{4}\right)$ is one of the promising materials for spin electronic applications due to its half-metallic nature, high Curie temperature, and a first order charge ordering transition at around $120 \mathrm{~K}$ (Verwey transition). ${ }^{1,2}$ These properties generated a renewed interest in studying the physical properties of epitaxial structures based on magnetite. Consequently, there are several reports on magnetic and transport property investigations on epitaxial films grown on a variety of substrates using various deposition methods. ${ }^{3-6}$ Differences in crystal symmetry and extent of lattice mismatch strain between $\mathrm{Fe}_{3} \mathrm{O}_{4}$ and substrate material are expected to influence the magnetic properties and even the band structure. The band structure of high temperature conducting phase of $\mathrm{Fe}_{3} \mathrm{O}_{4}$ is predicted to be half-metallic on the basis of first principles calculations based on the local density approximation (LDA), in which minority spin electrons are responsible for conduction. ${ }^{7,8}$ These band structure calculations give a magnetic moment of $4 \mu_{B} /$ f.u. for magnetite. However, some recent experimental results suggest that the itinerant electron picture for $\mathrm{Fe}_{3} \mathrm{O}_{4}$ is more adequate than the localized electron model. ${ }^{9,10}$ Despite rigorous efforts during the past decade, complete understanding of the nature of $3 d$ electronic correlations and complex band structure of magnetite are still a key issue.

High energy spectroscopies such as x-ray magnetic circular dichroism (XMCD) provide valuable information on the band structure of magnetic materials, as well as the site specific spin and orbital moments. ${ }^{11}$ The XMCD has been employed to investigate the spin structure of magnetite. In a

\footnotetext{
a) Author to whom correspondence should be addressed. Electronic mail: aroras@tcd.ie. FAX: +353-1-8963037.
}

recent investigation, Huang et al. ${ }^{12}$ reported a large unquenched $B$-site orbital moment of $0.33 \mu_{B}$, which has been attributed to a strong on-site Coulomb interaction and corresponding $3 d$ electron correlation effects. They obtained within the $\mathrm{LDA}+\mathrm{U}$ scheme similar values of unquenched orbital moments. However, further band structure calculation report only a small value of orbital moments. ${ }^{7,8}$ Contrary to the observation of Huang et al., Goering et al. ${ }^{13}$ reported nearly vanishing orbital moments for a $\mathrm{Fe}_{3} \mathrm{O}_{4}$ bulk crystal. We employ XMCD to investigate the nature of $3 d$ electrons in single crystalline $\mathrm{Fe}_{3} \mathrm{O}_{4}$ films. In this paper, we report the results on XMCD measurements performed in the soft x-ray absorption for epitaxial magnetite films on $\mathrm{MgO}$ (001) and $\mathrm{Al}_{2} \mathrm{O}_{3}$ (0001) substrates grown under identical conditions. Due to a small lattice mismatch, the films grown on $\mathrm{MgO}$ are fully strained, whereas the one grown on $\mathrm{Al}_{2} \mathrm{O}_{3}$ is fully strain relaxed owing to a larger lattice mismatch $(7.8 \%)$.

\section{EXPERIMENTAL}

Epitaxial $\mathrm{Fe}_{3} \mathrm{O}_{4}$ thin films of $100 \mathrm{~nm}$ thickness used in the present study were grown on $\mathrm{MgO}$ (001) and $\mathrm{Al}_{2} \mathrm{O}_{3}$ (0001) substrates under identical conditions using oxygen plasma assisted molecular beam epitaxy system (DCA MBE M600). The base pressure of the system is $5 \times 10^{-10}$ Torr. The growth of the films was carried out at a substrate temperature of $250{ }^{\circ} \mathrm{C}$. The details of the growth process are given elsewhere. ${ }^{14}$ Reflection high-energy electron diffraction (RHEED) was used to determine the growth mode and growth rate. The epitaxial relationship of both the heterostructures was established from the knowledge of in-plane $\left(a_{\|}\right)$and out-of-plane $\left(a_{\perp}\right)$ lattice parameters determined from $\omega-2 \theta$ rocking curves measured using a multicrystal high resolution x-ray diffractometer. Magnetization measure- 
ments were performed using a vibrating sample magnetometer (VSM) (PPMS-14T, Quantum Design, USA).

$\mathrm{X}$-ray absorption spectroscopy (XAS) was measured at the UE56/1-SGM beamline at the synchrotron light source BESSY II (Berlin) with perpendicularly incident photons and an external magnetic field of $1.0 \mathrm{~T}$ applied parallel to the photon beam. The $\mathrm{x}$-ray luminescence in the substrate ${ }^{15}$ measures the photon flux transmitted through the film [transmission (TM) signal]. This information reflects the film properties integrated along the film normal. The simultaneously measured total electron yield (TEY) via the sample current serves as surface sensitive information with a probing depth of $0.8 \mathrm{~nm}$ for $\mathrm{Fe}_{3} \mathrm{O}_{4} \cdot{ }^{13}$ For XMCD, we take the difference of spectra recorded with opposite magnetization direction.

\section{RESULTS AND DISCUSSIONS}

Prior to discussing the results of magnetic circular dichroism on these films, we would like to point out that the films are highly stoichiometric, as determined from in situ RHEED and ex situ high resolution x-ray diffraction measurements. From the analysis of symmetric $(200 / 400)$ and asymmetric (311/622) Bragg diffraction for the $\mathrm{Fe}_{3} \mathrm{O}_{4} / \mathrm{MgO}(001)$ heteroepitaxial system, we find that the films are fully strained. ${ }^{14}$ The values of in-plane and out-of plane lattice parameters for $\mathrm{Fe}_{3} \mathrm{O}_{4}$ film on $\mathrm{MgO}$ being 0.8426 and $0.8372 \mathrm{~nm}$, respectively. Similar investigations for films on $\mathrm{Al}_{2} \mathrm{O}_{3}$ (0001) substrates suggest that the film was almost completely strain relaxed (out-of-plane lattice parameter for film is $0.8378 \mathrm{~nm}$ ) in the sense that most of the epitaxial lattice mismatch strain has relaxed. This is due to the greater lattice mismatch of $\mathrm{Fe}_{3} \mathrm{O}_{4}$ with $\mathrm{Al}_{2} \mathrm{O}_{3}(7.8 \%$, compressive) as compared to $\mathrm{MgO}(0.33 \%$, tensile). Yet, it should be noticed that even $90 \%$ strain-relaxed film $\mathrm{Fe}_{3} \mathrm{O}_{4} / \mathrm{Al}_{2} \mathrm{O}_{3}$ has still more strain than completely unrelaxed $\mathrm{Fe}_{3} \mathrm{O}_{3} / \mathrm{MgO}$ film. In addition to the structural characterization, the presence of the Verwey transition in our samples was used as a further affirmation to the fact that the films are highly stoichiometric, as this transition is very sensitive to the deviation from the stoichiometry. Figure 1 shows the magnetization for both the samples as a function of temperature measured in the presence of 200 Oe field. The structural change at the Verwey transition invokes changes of the magnetization state presumably caused by a decreased magnetic anisotropy. The increase of the observed moment sensitively detects the change of the magnetization state at the transition temperature. It is clear that both films exhibit the Verwey transition. The Verwey transition temperature $\left(T_{V}\right)$ is $123 \mathrm{~K}$ for the film on $\mathrm{Al}_{2} \mathrm{O}_{3}$, whereas its value is $119 \mathrm{~K}$ for the film on $\mathrm{MgO}$.

We show a typical XAS and XMCD spectrum in Fig. 2(a). The general shape of the XAS spectra is comparable to the results presented for bulk $\mathrm{Fe}_{3} \mathrm{O}_{4} \cdot{ }^{12,13}$ The discussion of minor differences between the TEY and TM XAS spectra and between spectra measured below and above $T_{V}$ exceeds the scope of this article. The integrated XMCD signal [Fig. 2(b)] exhibits a nonvanishing slope between 730 and $765 \mathrm{eV}$. The nonvanishing slope indicates a small and reproducible XMCD signal above the $L_{2}$ edge region, similar to the one observed in Ref. 13.

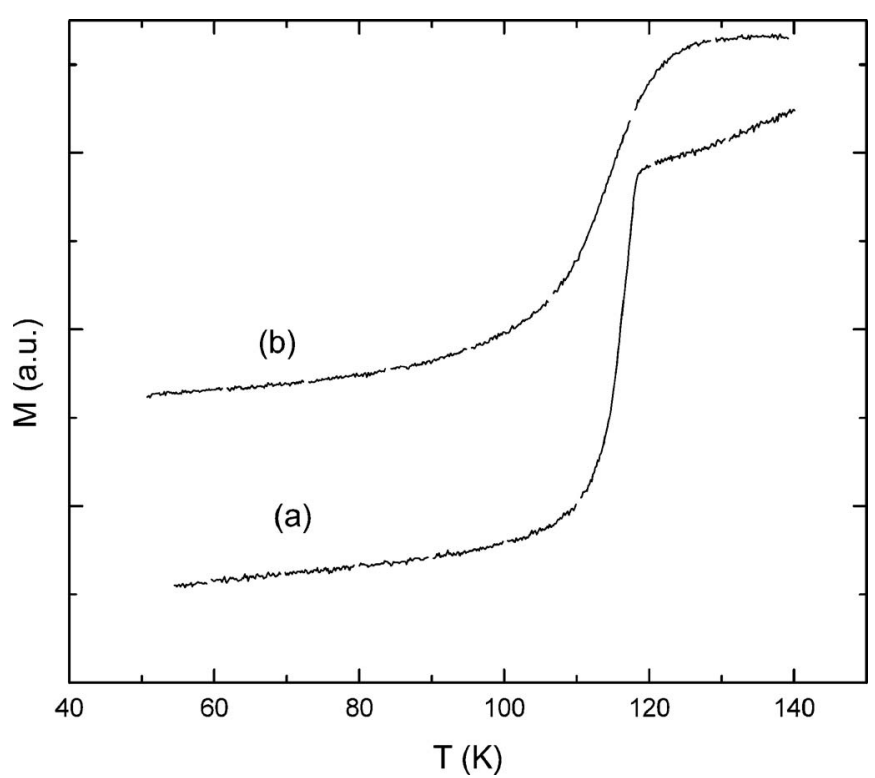

FIG. 1. Magnetization as a function of temperature for the $100 \mathrm{~nm} \mathrm{Fe} \mathrm{O}_{4}$ films on (a) $\mathrm{MgO}$ (001) and (b) $\mathrm{Al}_{2} \mathrm{O}_{3}$ (0001) substrates. The magnetization was measured in a 200 Oe field during the warming cycle after cooling the sample from room temperature to $50 \mathrm{~K}$ in zero field.

We have applied sum rules for the $L_{2}$ edge integration range of 718-770 eV. The two-step background signal $\mu_{\mathrm{bg}}$ that has been subtracted is shown in Fig. 2(a). For the number of $\mathrm{Fe} 3 d$ holes, we assumed a value of $N_{h} / \mathrm{Fe}=13.5 / 3$, identical to the value used in Ref. 13. Consideration of selfabsorption effects is not applicable for TM XAS and can be neglected in the case of TEY XAS. ${ }^{13}$ Results are shown in Table I. For the orbital to spin moment ratio $\mu_{\text {spin }} / \mu_{\text {orb }}$, the estimated error bar reflects data noise and uncertainties due to the integration range. For the effective spin moment $\mu_{\text {spin }}=\left(\mu_{s}+7\left\langle T_{z}\right\rangle\right)$, an additional error arising from the uncertainty of the background subtraction must be considered. Ad-

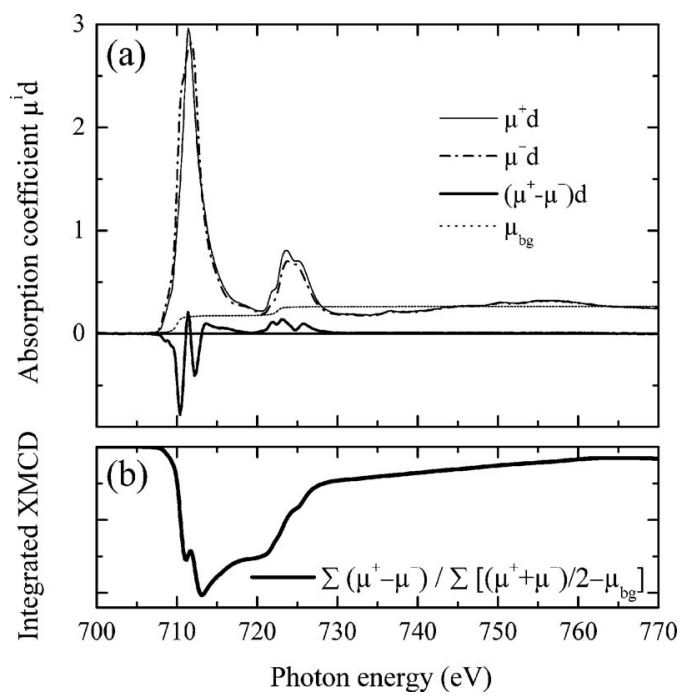

FIG. 2. (a) X-ray absorption coefficients $\mu^{i}$ for magnetization parallel and antiparallel to the $\mathrm{x}$-ray polarization vector multiplied by the film thickness $d$ and XMCD spectrum $\mu^{+}-\mu^{-}$of a $100 \mathrm{~nm} \mathrm{Fe}_{3} \mathrm{O}_{4}$ film on $\mathrm{Al}_{2} \mathrm{O}_{3}(0001)$ at $300 \mathrm{~K}$ (normal-incidence transmission geometry). (b) Integrated XMCD spectrum used for sum rule analysis. 
TABLE I. Effective magnetic spin moment per Fe atom and orbital to spin moment ratio as determined by the sum rules from the XMCD spectra. Total Fe moment recalculated from the saturation magnetization as determined by VSM for the same samples are given for comparison. Values in parenthesis indicate the error bar.

\begin{tabular}{|c|c|c|c|c|c|}
\hline & $\begin{array}{c}77 \mathrm{~K} \\
\mu_{\text {spin }}\left(\mu_{B}\right)\end{array}$ & $\begin{array}{c}77 \mathrm{~K} \\
\mu_{\text {orb }} / \mu_{\text {spin }}\end{array}$ & $\begin{array}{c}300 \mathrm{~K} \\
\mu_{\text {spin }}\left(\mu_{B}\right)\end{array}$ & $\begin{array}{c}300 \mathrm{~K} \\
\mu_{\mathrm{orb}} / \mu_{\text {spin }}\end{array}$ & $\begin{array}{c}300 \mathrm{~K} \\
\mu_{\mathrm{VSM}}\left(\mu_{B}\right)\end{array}$ \\
\hline \multicolumn{6}{|c|}{$\mathrm{Fe}_{3} \mathrm{O}_{4} / \mathrm{MgO}$} \\
\hline $\mathrm{TM}$ & $0.86(2)$ & $0.01(1)$ & $0.74(13)$ & $0.03(3)$ & 1.36 \\
\hline TEY & $0.99(2)$ & $0.00(2)$ & $0.99(2)$ & $0.04(2)$ & \\
\hline \multicolumn{6}{|c|}{$\mathrm{Fe}_{3} \mathrm{O}_{4} / \mathrm{Al}_{2} \mathrm{O}_{3}$} \\
\hline $\mathrm{TM}$ & $0.89(2)$ & $0.01(1)$ & $0.91(2)$ & $0.025(10)$ & 1.2 \\
\hline TEY & $0.86(2)$ & $0.06(2)$ & $0.87(2)$ & $0.04(2)$ & \\
\hline
\end{tabular}

ditional systematic errors (same factor for all values given in Table I) due to the finite $x$-ray polarization and to the unknown exact value of $N_{h}$ were neglected.

Only a minor temperature dependency of the spin moment is observed, in agreement with the saturation magnetization data from VSM measurements and comparable with data from a bulk $\mathrm{Fe}_{3} \mathrm{O}_{4}$ crystal. ${ }^{13}$ The saturation magnetization of the films (see Table I) is in agreement with the bulk value for $\mathrm{Fe}_{3} \mathrm{O}_{4}$. However, $\mu_{\text {spin }}$ measured by XMCD is for both films about $30 \%$ smaller than the saturation magnetization measured for these samples by VSM magnetometry. We attribute this discrepancy to the error in determining the moment from VSM measurements and partly to the finite x-ray polarization.

A remarkable result is the increase of the surface spin moment of $\mathrm{Fe}_{3} \mathrm{O}_{4} / \mathrm{MgO}$ with respect to the bulk value of the film. This fact might be attributed to the smaller average coordination number for atoms at the surface. An increased surface moment was not observed in the case of $\mathrm{Fe}_{3} \mathrm{O}_{4} / \mathrm{Al}_{2} \mathrm{O}_{3}$, indicating a counteracting influence of antiphase boundaries.

It is also remarkable that the difference between surface and bulk moments is comparatively small despite the fact that the samples have been stored in air for several days. This observation indicates that the surface of the $\mathrm{Fe}_{3} \mathrm{O}_{4}$ films does not react considerably with oxygen at ambient conditions, thus implying a promising precondition for industrial processing.

The average orbital moments are much smaller than those reported in Ref. 12. At $77 \mathrm{~K}$, the bulk values of both films are only $0.01 \mu_{B}$, confirming the vanishing orbital moment contribution found for a bulk $\mathrm{Fe}_{3} \mathrm{O}_{4}$ crystal. ${ }^{13}$ At $300 \mathrm{~K}$, the orbital moment has increased to $0.03 \mu_{B}$. This might be attributed to the thermally induced epitaxial strain. An increase of the orbital moment at the surface is not significant except the low-temperature TEY value of the
$\mathrm{Fe}_{3} \mathrm{O}_{4} / \mathrm{Al}_{2} \mathrm{O}_{3}$ (0001) film. In general, a larger surface orbital moment could be expected because of decreased crystal symmetry at the surface.

In summary, we determined element-specific magnetic spin and orbital moments at the surface and in the bulk of epitaxial $\mathrm{Fe}_{3} \mathrm{O}_{4}$ films in two orientations. Magnetization measurements at low field clearly confirm the presence of a Verwey transition in the films. The saturation magnetization is close to reference values found for a bulk $\mathrm{Fe}_{3} \mathrm{O}_{4}$ crystal, thus confirming the good quality of the investigated films. At $77 \mathrm{~K}$, we found an increased surface spin moment for $\mathrm{Fe}_{3} \mathrm{O}_{4} / \mathrm{MgO}(001)$ and an increased surface orbital moment for $\mathrm{Fe}_{3} \mathrm{O}_{4} / \mathrm{Al}_{2} \mathrm{O}_{3}(0001)$.

${ }^{1}$ M. Ziese, Rep. Prog. Phys. 65, 143 (2002).

${ }^{2}$ F. Waltz, J. Phys.: Condens. Matter 14, R285 (2002).

${ }^{3}$ W. Eerenstein, T. T. M. Palstra, T. Hibma, and S. Celotto, Phys. Rev. B 68, 014428 (2003)

${ }^{4}$ D. T. Margulies, F. T. Parker, F. E. Spada, R. S. Goldman, J. Li, R. Sinclair, and A. E. Berkowitz, Phys. Rev. B 53, 9175 (1996).

${ }^{5}$ S. Kale, S. M. Bhagat, S. E. Loafland, T. Scabarozi, S. B. Ogale, A. Orozco, S. R. Shinde, T. Venkatesan, B. Hannoyer, B. Mercey, and W. Prellier, Phys. Rev. B 64, 205413 (2001).

${ }^{6}$ S. K. Arora, R. G. S. Sofin, and I. V. Shvets, Phys. Rev. B 72, 134404 (2005).

${ }^{7}$ H. T. Cheng and G. Y. Guo, Phys. Rev. B 65, 094429 (2002).

${ }^{8}$ Z. Zhang and S. Satpathy, Phys. Rev. B 44, 13319 (1991).

${ }^{9}$ P. Novak, H. Stepankova, J. Englich, J. Kohout, and V. A. M. Barbers, Phys. Rev. B 61, 1256 (2000).

${ }^{10}$ J. Garcia, G. Subias, M. G. Proietti, H. Renevier, Y. Joly, J. L. Hodeau, J. Blasco, M. C. Sanchez, and J. F. Berar, Phys. Rev. Lett. 85, 578 (2000).

${ }^{11}$ J. Stohr, J. Magn. Magn. Mater. 200, 470 (1999).

${ }^{12}$ D. J. Huang, C. F. Chang, H.-T. Jeng, G. Y. Guo, H.-J. Lin, W. B. Wu, H. C. Ku, A. Fujimori, Y. Takahashi, and C. T. Chen, Phys. Rev. Lett. 93, 077204 (2004)

${ }^{13}$ E. Goering, S. Gold, M. Lafkioti, and G. Schutz, Europhys. Lett. 73, 97 (2006).

${ }^{14}$ S. K. Arora, R. G. S. Sofin, I. V. Shvets, and M. Luysberg, J. Appl. Phys. 100, 073908 (2006).

${ }^{15}$ M. Kallmayer, H. Schneider, G. Jakob, H. J. Elmers, K. Kroth, H. C. Kandpal, U. Stumm, and S. Cramm, Appl. Phys. Lett. 88, 072506 (2006). 